\title{
Características Físico-Químicas, Organolépticas y Reológicas de Arepas Procesadas a partir de Maíces de Alta Calidad Proteica (QPM)
}

\author{
Paula Andrea Marín* \\ Carlos de León** \\ Harold Acosta Z.**
}

* Ingeniera Química, Universidad del Valle. e-mail: paulam94@yahoo.com

** Investigador Centro Internacional de Mejoramiento de Maíz y Trigo, México (Sede CIAT, Palmira, Colombia). e-mail: c.deleon@cgiar.org

*** Departamento de Ingeniería de Alimentos. Universidad del Valle. e-mail: hacostaz@yahoo.com, hacostaz@univalle.edu.co

Fecha de recepción: Junio 13 de 2003

Fecha de aprobación: Octubre 17 de 2003

\section{RESUMEN}

Se obtuvieron masas de cinco variedades de maíz (QPM Blanco, 'QPMB'; QPM Amarillo, 'QPMA'; Híbrido A.M., 'HAM'; Pab SA4, 'PS4'; y Maíz Común, MC) usando un método de laboratorio. El mayor rendimiento durante la cocción y las masas más suaves se obtuvieron con $17 \mathrm{~h}$ de remojo y $4 \mathrm{~h}$ de cocción. Grasa y almidón fueron semejantes para QPMB y MC, el cual mostró valores muy bajos de humedad y proteína, las demás variedades tuvieron valores semejantes. HAM y PS4 fueron catalogados como maíces altos en grasa. Las características físicas de HAM y QPMA fueron similares a MC. Tres variedades se clasificaron como Grado 3 (maíz tipo exportación). Arepas hechas con estas variedades fueron aceptadas 
sensorialmente (color, sabor y aroma) en el orden: $\mathrm{QPMB}>\mathrm{CM}>\mathrm{QPMA}>\mathrm{HAM}>\mathrm{Ps} 4$.

Se hicieron pruebas reológicas, mostrando que las arepas de las cinco variedades, se clasificaron como frágiles, según sus curvas de compresión. La inclinación de la curva en la sección elástica fue similar en todos los casos, indicando maleabilidad similar. Las arepas de MC y QPMB fueron las más blandas, al requerir la menor fuerza para alcanzar la máxima compresión. Las curvas reológicas de extrusión, mostraron que las arepas de las cinco variedades de maíz, se caracterizaron por poseer firmeza, cohesividad y adhesividad semejantes.

Palabras Clave: Maíz de alta calidad proteica, QPM, reología de masas, análisis sensorial, caracterización físico-química de masas, balance de masa.

\section{ABSTRACT}

Masas from five maize varieties (White QPM, 'QPMB'; Yellow QPM, 'QPMA'; Hybrid A.M., 'HAM'; Pab SA4, 'PS4'; and Common Maize, MC) were obtained using a lab scale method. The best cooking yield and the smoothest masas were produced when all maizes were soaked for $17 \mathrm{~h}$ and cooked for $4 \mathrm{~h}$. Fat and starch were similar for QPMB and MC, but the latter had lower protein and moisture values compared to the other varieties. HAM and PS4 were classified as high oil varieties. HAM, QPMA and MC had similar physical characteristics. Three varieties were catalogued as Grade 3 (export quality maize).

Arepas made from these masas were sensorially acceptable according to color, flavor and aroma in this order: QPMB > CM > QPMA > HAM > PS4.

Rheological tests were performed, showing that arepas from the five varieties were classified as fragile, according to their Compression tests. The slope of the elastic portion of the compression curve was similar for all varieties, indicating equivalent malleability. Arepas made from $M C$ and QPMB were the softest, requiring less strength to attain maximum compression. Rheological extrusion curves indicated that arepas from the five varieties had similar firmness, cohesivity, and adhesivity.
Key Words: Quality Protein Maize, QPM, rheology of masas, sensory analysis, masa physico-chemical characterization, mass balance.

\section{INTRODUCCIÓN}

El maíz, un alimento básico en los países en desarrollo, ha impulsado investigaciones para mejorar su valor nutritivo. ${ }^{8}$ Inicialmente, se descubrió un maíz de elevada calidad proteica, que se denominó Opaco 2q, pero de pobres propiedades agronómicas.' Los fitomejoradores trabajaron por 30 años para obtener híbridos de alta proteína (conocidos como QPM O 'Quality Protein Maize') con características agronómicas comerciales. ${ }^{5,10}$

Por esto, se planteó la necesidad de determinar características físico-químicas, organolépticas y reológicas de maíces y de arepas (masas de maíz asadas, muy comunes en Colombia y Venezuela) elaboradas a partir de maíces de alta proteína y compararlas con las de otros maíces. También, se realizaron los balances de materia para el proceso de producción de arepas.

\section{MATERIALES Y MÉTODOS}

\section{Materiales}

Se usaron cinco variedades de maíz: común (CM, usada como referencia), Híbrido A.M. (HAM), QPM Blanco (QPMB), QPM Amarillo (QPMA) y PabSA4 (PS4), provenientes de los experimentos llevados a cabo por CIMMYT-CIAT en el Departamento de Córdoba durante 2002. Se realizaron muestreos aleatorios de granos cultivados en varias áreas, que luego fueron combinados en una muestra compuesta para simular las que usualmente se comercializan. Las pruebas se hicieron mínimo en triplicado y los resultados se analizaron usando un diseño completamente aleatorio, empleando el programa MINITAB.

\section{Características físico-químicas}

Se determinaron humedad, grasa, proteína, almidón total, densidad, fragilidad, peso de mil granos, peso hectolítrico, porcentaje de granos 
dañados y las dimensiones, siguiendo métodos AACC. ${ }^{1,2,3}$ Todas las determinaciones se reportaron en base seca.

\section{Elaboración de arepas y balance de materia}

El balance de materia se realizó en cada etapa del proceso de elaboración de arepas.

Se pesaron $150 \mathrm{~g}$ de maíz, se adicionó 1 L de agua y se remojaron por $17 \mathrm{~h}$, buscando la máxima hidratación. Luego, se cocinaron durante 3 h, se escurrió el maíz sin descartar el agua y se pesó el maíz cocido, se molió en un molino manual, se pesó la masa obtenida en la molienda y se amasó. Al final, se armaron las arepas con un molde, se pesaron, se asaron en plancha por 8 min y se pesaron después de asadas.

\section{Características organolépticas}

Las características organolépticas (color, sabor y aroma de la arepa) se determinaron siguiendo la escala de la Tabla 1 para una prueba hedónica (de la cual se aprovechó su característica de evaluar globalmente el producto).

\begin{tabular}{|l|c|}
\hline \multicolumn{1}{|c|}{ DESCRIPCIÓM } & CALIFICACIÓM \\
\hline Gusta Muchísimo & 9 \\
\hline Gusta Mucho & 8 \\
\hline Gusta Moderadamente & 7 \\
\hline Gusta un poco & 6 \\
\hline Es indiferente & 5 \\
\hline Disgusta un poco & 4 \\
\hline Disgusta Moderadamente & 3 \\
\hline Disgusta Mucho & 2 \\
\hline Disgusta Muchísimo & 1 \\
\hline
\end{tabular}

Tabla 1. Escala Hedónica

La prueba se realizó con un panel de ocho jueces no entrenados, pero con experiencia en degustar este plato típico; la evaluación de cada producto se hizo por separado. Las arepas se elaboraron el mismo día de la prueba y fueron presentadas en cinco bandejas, una por variedad de maíz.

\section{Características reológicas}

Las características reológicas de compresión y de extrusión (de arepas sin asar, pues la masa es la que sufre más alteraciones) ${ }^{6}$, se realizaron con una Máquina Universal de Ensayos, IMSTROM. Se realizaron pruebas en arepas elaboradas el mismo día para evitar alteración del producto. Se calibró la INSTROM a cero absoluto y las pruebas se realizaron a una velocidad de operación de 5 $\mathrm{mm} / \mathrm{min}$ y $10 \mathrm{~mm}$ de recorrido. Se instaló una placa de diámetro superior al de la sección de la arepa para el análisis de compresión y una rejilla para el de extrusión. ${ }^{4}$

\section{RESULTADOS Y DISCUSIÓN}

\section{Análisis físico-químico}

El análisis físico-químico se presentan en la Tabla 2. La prueba de fragilidad en un Ro-Tap mostró que todos los granos de todas las muestras quedaron en la bandeja superior sin quebrarse, indicando que el maíz se encuentra en buen estado y que tiene una buena resistencia mecánica al impacto. ${ }^{6,7}$

La resistencia mecánica del grano se debe a que se controlaron factores como contenidos muy bajos de humedad, bajo impacto contra superficies duras durante el transporte $y$ métodos de secado no apropiados. ${ }^{7,10}$

\begin{tabular}{|c|c|c|c|c|c|}
\hline PROPIEDAD & MC & HAM & QPMB & QPMA & Ps4 \\
\hline $\begin{array}{c}\text { Peso de mil granos } \\
(\mathrm{g})[\mathrm{s}]\end{array}$ & 345.4 & 319.7 & 250.1 & 354.7 & 387.4 \\
{$[2.47]$} & {$[2.92]$} & {$[1.58]$} & {$[3.11]$} & {$[3.82]$} \\
\hline $\begin{array}{c}\text { Peso hectolítrico } \\
\text { (g) }[\mathrm{s}]\end{array}$ & 192.0 & 189.3 & 185.9 & 181.7 & 188.3 \\
{$[1.03]$} & {$[1.98]$} & {$[1.74]$} & {$[1.87]$} & {$[1.58]$} \\
\hline $\begin{array}{c}\text { Granos dañados } \\
(\%)[\mathrm{s}]\end{array}$ & 2.5 & 3.4 & 2.7 & 2.5 & 3.7 \\
{$[0.47]$} & {$[0.75]$} & {$[0.63]$} & {$[0.42]$} & {$[0.56]$} \\
\hline $\begin{array}{c}\text { Densidad } \\
(\text { Kg/m3) }[\mathrm{s}]\end{array}$ & 768.1 & 757.2 & 743.8 & 726.6 & 753.1 \\
\hline$[5.12]$ & {$[4.75]$} & {$[4.69]$} & {$[4.98]$} & {$[5.23]$} \\
\hline $\begin{array}{c}\text { Ancho } \\
(\mathrm{mm})[\mathrm{s}]\end{array}$ & 9.1 & 8.8 & 8.6 & 9.0 & 9.5 \\
{$[0.96]$} & {$[1.14]$} & {$[1.26]$} & {$[1.06]$} & {$[1.17]$} \\
\hline $\begin{array}{c}\text { Largo } \\
(\mathrm{mm})[\mathrm{s}]\end{array}$ & 11.8 & 10.8 & 9.5 & 11.7 & 12.2 \\
{$[1.13]$} & {$[1.20]$} & {$[1.08]$} & {$[1.11]$} & {$[1.21]$} \\
\hline $\begin{array}{c}\text { Espesor } \\
(\mathrm{mm})[\mathrm{s}]\end{array}$ & 4.3 & 4.8 & 4.1 & 4.8 & 4.6 \\
{$[0.45]$} & {$[0.98]$} & {$[0.91]$} & {$[0.77]$} & {$[0.87]$} \\
\hline
\end{tabular}

MC: maíz común; HAM: Híbrido AM; QPMB: QPM Blanco; QPMA: QPM Amarillo; Ps4: PabSA4; [s]: desviación estándar

Tabla 2. Características Físicas de Variedades de Maíz 
Comparando los pesos de mil granos y peso hectolítrico obtenidos, con la norma estándar de los Estados Unidos para determinar el valor comercial, se encontró que estas variedades se clasificaban entre grado 2 y $4 .{ }^{10}$ EI MC se clasificó en el grado 2 y el QPMA en grado 4; las otras tres variedades correspondían al grado 3 (grado comercial mínimo para exportación). ${ }^{7,10}$

El MC al igual que el QPMB y el QPMA presentaron daño por fisuras, por granos quebrados y ataque de insectos, mientras que no se registró ataque de hongos. La principal causa de daño en estas variedades fue la presencia de fisuras, aunque esto no tuvo incidencia en la prueba de fragilidad donde se encontró que poseían buena resistencia. De acuerdo a la norma estándar, se permite hasta un $3.0 \%$ de granos dañados en el grado 3, por tanto estas variedades son comerciables, excepto HAM y PS4, que están un poco por encima del máximo. ${ }^{7,10}$

La densidad presentó un valor medio de 742.5 $\mathrm{Kg} / \mathrm{m}^{3}$. Esta prueba indica el nivel de nutrientes digeribles del maíz, a mayor densidad mayor contenido de nutrientes. Un maíz se considera de baja densidad si está debajo de $650.0 \mathrm{~kg} / \mathrm{m}^{3}$, en este caso todas las variedades se encuentran por encima de este valor, indicando variedades de buen contenido de nutrientes. ${ }^{10}$

La variedad con mayor tamaño de grano fue el PS4, sus dimensiones (ancho, largo y espesor) fueron las más altas dentro del grupo y la variedad con menor tamaño fue el QPMB. La variedad que más se asemejó al maíz común fue el QPMA.

El contenido de humedad de las diferentes muestras osciló entre el 8.2 y $11.3 \%$ valores aceptables para conservar el grano durante el almacenamiento, sin deterioro microbiológico. ${ }^{10}$ El maíz más seco fue el común y el que presentó mayor contenido de humedad fue el QPMA. El contenido de humedad depende básicamente del método de secadoutilizado. ${ }^{7}$

La variedad con menor contenido de extracto etéreo (\% grasa) fue el común que junto con el QPMB presentaron 5.5\%, seguida del QPMA con $6.0 \%$ y las variedades con mayor contenido fueron el HAM y el PS4 con $7.7 \%$ y $7.3 \%$, respectivamente. El contenido de grasa estuvo dentro del requerimiento comercial (por encima del $4.4 \%$ ). Las variedades HAM y PS4 se pueden considerar como "maíces altos en grasa" y con valor comercia l extra. ${ }^{10}$

\begin{tabular}{|c|c|c|c|c|c|}
\hline PROPIEDAD & MC & HAM & QPMB & QPMA & Ps4 \\
\hline $\begin{array}{c}\text { Humedad } \\
(\%)[s]\end{array}$ & $\begin{array}{c}8.2 \\
{[1.23]}\end{array}$ & $\begin{array}{c}9.3 \\
{[1.03]}\end{array}$ & $\begin{array}{l}10.2 \\
{[1.30]}\end{array}$ & $\begin{array}{c}11.3 \\
{[1.41]}\end{array}$ & $\begin{array}{c}11.2 \\
{[1.05]}\end{array}$ \\
\hline $\begin{array}{c}\text { Grasa } \\
(\%)[s]\end{array}$ & $\begin{array}{c}5.5 \\
{[0.72]}\end{array}$ & 7.7 & 5.5 & 6.0 & 7.3 \\
{$[0.69]$} & {$[0.68]$} & {$[0.75]$} & {$[0.79]$} \\
\hline $\begin{array}{c}\text { Proteína } \\
(\%)[s]\end{array}$ & 10.8 & 12.9 & 13.3 & 13.1 & 13.8 \\
{$[1.28]$} & {$[1.33]$} & {$[1.37]$} & {$[1.04]$} & {$[1.56]$} \\
\hline $\begin{array}{c}\text { Almidón total } \\
(\%)[s]\end{array}$ & 62.5 & 59.8 & 59.8 & 54.0 & 57.0 \\
{$[3.51]$} & {$[3.96]$} & {$[3.96]$} & {$[3.67]$} & {$[2.76]$} \\
\hline
\end{tabular}

Clave: MC: maíz común; HAM: Híbrido AM; QPMB: QPM Blanco; QPMA: QPMA Amarillo; Ps4: PabSA4; [s]: desviación estándar

Tabla 3. Composición Química de Variedades de Maíz

La proteína de las cinco variedades osciló entre $10.8 \%$ y $13.8 \%$ correspondientes a maíz común y P54 respectivamente. La proteína en variedades comunes puede oscilar entre el $8.0 \%$ y el $11.0 \%$, con algunas variedades hasta $14.0 \%$. En este caso, el contenido está dentro de parámetros normales. ${ }^{4}$ Sin embargo, esto no se relaciona con la calidad proteica del grano, pues no cuenta la cantidad de proteína del grano, sino los aminoácidos constituyentes. Las variedades QPM presentaron un buen contenido proteico comparado con el común y si a esto se le suma el que una fracción de zeína es ocupada por lisina y triptófano, entonces se obtiene un producto que aporta al nivel nutricional una buena dosis de proteínas de alta calidad. ${ }^{5,7,10}$

El contenido de almidón total osciló entre el $53.7 \%$ y $62.5 \%$ del maíz HAM y MC respectivamente, mostrando que algunos valores están debajo de los comerciales (mínimo $60.0 \%) .{ }^{10}$ 


\section{Balances de materia}

El balance de materia se hizo con el fin de conocer el rendimiento del maíz durante el proceso de elaboración de arepas. Se hicieron cuatro ensayos con cada una de las variedades de maíz en los que se variaron los tiempos de cocción y de remojo:

- Ensayo 1: $\mathrm{Tr}=17$ y $\mathrm{Tc}=3 \mathrm{~h}$

- Ensayo 2: $\mathrm{Tr}=17$ y $\mathrm{Tc}=4 \mathrm{~h}$

- Ensayo 3: $\operatorname{Tr}=0 \mathrm{~h}$ y $\mathrm{Tc}=3 \mathrm{~h}$

- Ensayo 4: $\mathrm{Tr}=0 \mathrm{~h}$ y $\mathrm{Tc}=4 \mathrm{~h}$

(Tr $=$ tiempo de remojo y $\mathrm{Tc}=$ tiempo de cocción).

PS4 fue la variedad con menor rendimiento durante la cocción, este comportamiento fue igual en los diferentes ensayos (Fig. 1). Este bajo rendimiento se puede explicar por su endospermo duro, que requiere mayor tiempo de cocción para absorber el agua necesaria para que el grano se hinche lo suficiente y gane peso. Ocurre lo contrario con MC, el cual en los ensayos 1,2 y 4 presentó los más altos rendimientos entre las cinco variedades; resultando, ensayo 1 : $287.3 \%$, ensayo 2: $288.0 \%$ y ensayo $4: 284.0 \%$; ensayo 3: $253.0 \%$, el cual mostró un comportamiento diferente.

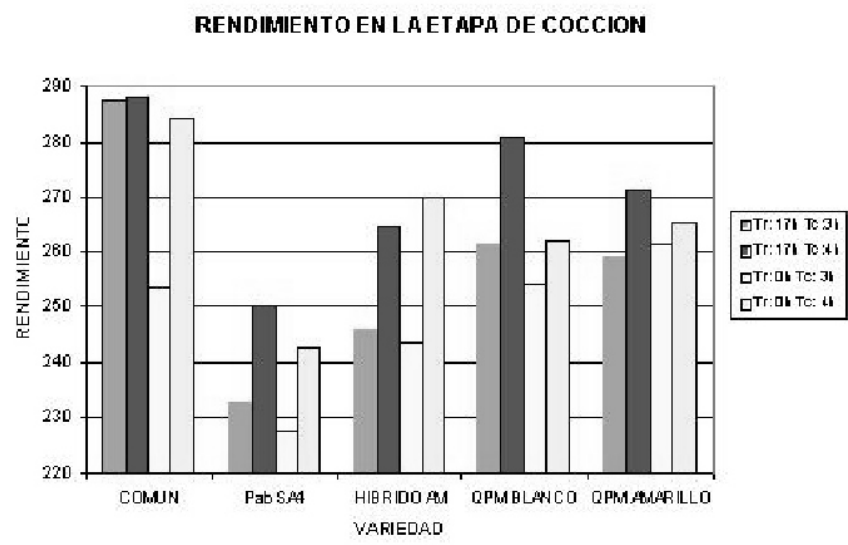

Figura 1. Comparación del rendimiento de variedades de maíz, usando cuatro métodos de preparación, variando el tiempo de remojo (Tr) y el tiempo de cocción (Tc)

Se usó un molino manual de tornillo sin fin en la molienda; la masa obtenida presentó mucho afrecho, pues el ensayo se hizo con maíz entero. Masas de 3 h de cocción, fueron secas y difíciles de amasar; mientras que masas de $4 \mathrm{~h}$ de cocción, fueron suaves y maleables. Esto muestra que el mayor tiempo de cocción, permite que el grano absorba más agua y se logre un mayor ablandamiento, pero tiempos mayores pueden dar masas pegajosas. Las masas se moldearon, obteniendo arepas con peso promedio de $73.0 \mathrm{~g}( \pm 2.1)$, las cuales perdieron entre $11 \%$ y $14 \%$ de peso (agua evaporada) durante el asado de $8 \mathrm{~min}$ a que fueron sometidas.

\section{Características organolépticas}

El color, el sabor y el aroma de las arepas obtenidas de cada variedad, mostraron un comportamiento similar al maíz común, el más usado en Colombia.

Los niveles de aceptación del color, sabor y aroma de acuerdo con la evaluación de los panelistas, se muestran en las Tablas 4, 5 y 6, basados en la escala hedónica.

El sabor de las arepas de las cinco variedades tuvieron diferente aceptación, mostrando que están por encima del valor neutral (5.0) y que los maíces de alta proteína, presentan la mejor aceptación. ${ }^{9}$

\begin{tabular}{l|c|c|}
\hline \multicolumn{1}{c|}{ VARIEDAD } & VALOR & DESCRIPCIÓM \\
\hline Común & $7.0[0.91]$ & Gusta Moderadamente \\
\hline Híbrido A.M. & $6.1[0.78]$ & Gusta Poco \\
\hline PABSA4 & $5.1[0.89]$ & Indiferente \\
\hline QPM Amarillo & $7.2[0.42]$ & Gusta Moderadamente \\
\hline QPM Blanco & $8.2[0.52]$ & Gusta Mucho \\
\hline
\end{tabular}

Clave: MC: maíz común; HAM: Híbrido AM; QPMB: QPM Blanco; QPMA: QPM Amarillo; PS4: PabSA4. Los valores entre corchetes se refieren a su desviación estándar

Tabla 4. Valoración del sabor de arepas hechas de variedades de maíz

Las arepas que obtuvieron la mejor aceptación en esta prueba fueron las procesadas a partir de maíz QPMB y MC, con calificación encima de 9 puntos. En todos los casos, las calificaciones fueron superiores a 7.0, lo que indica que el aroma de las arepas de las cinco variedades estudiadas fueron aceptables. 


\begin{tabular}{|l|c|c|}
\hline \multicolumn{1}{|c|}{ VARIEDAD } & VALOR & DESCRIPCIÓM \\
\hline Común & $9.0[0.89]$ & Gusta Muchísimo \\
\hline Híbrido A.M. & $8.2[0.98]$ & Gusta Mucho \\
\hline PABSA4 & $7.2[1.02]$ & Gusta Moderadamente \\
\hline QPM Amarillo & $7.1[0.68]$ & Gusta Moderadamente \\
\hline APM Blanco & $9.1[1.03]$ & Gusta Muchísimo \\
\hline
\end{tabular}

Clave: MC: maíz común; HAM: Híbrido AM; QPMB: QPM Blanco; QPMA: QPM Amarillo; PS4: PabSA4. Los valores entre corchetes se refieren a su desviación estándar

Tabla 5. Valoración del aroma de arepas hechas de variedades de maíz

\begin{tabular}{|l|c|c|}
\hline \multicolumn{1}{|c|}{ VARIEDAD } & VALOR & DESCRIPCIÓM \\
\hline Común & $9.1[0.25]$ & Gusta Muchísimo \\
\hline Híbrido A.M. & $8.3[0.28]$ & Gusta Mucho \\
\hline PABSA4 & $7.2[0.39]$ & Gusta Moderadamente \\
\hline QPM Amarillo & $9.0[0.32]$ & Gusta Muchísimo \\
\hline QPM Blanco & $9.0[0.38]$ & Gusta Muchísimo \\
\hline
\end{tabular}

Clave: MC: maíz común; HAM: Híbrido AM; QPMB: QPM Blanco; QPMA: QPM Amarillo; PS4: PabSA4. Los valores entre corchetes se refieren a su desviación estándar

Tabla 6. Valoración del color de arepas hechas de variedades de maíz

El color de las arepas fueron calificadas entre 8.0 y 9.0, excepto para P54. Los maíces QPM fueron calificados en el grado más alto de aceptación ('gusta muchísimo'). Estos resultados muestran que los maíces QPM presentan desempeño en producción y aceptación organoléptica, comparable con MC, por lo cual se podría implementar su cultivo para suplir las necesidades de seguridad alimentaria y de calidad nutricional de una población dependiente de este plato tradicional.

\section{Reología.}

Pruebas reológicas de compresión de arepas sin asar. En la Tabla 7 se presenta la pendiente de la región elástica de las curvas obtenidas en la máquina IMSTROM.

La inclinación en la región elástica fue muy pequeña, lo cual era de esperarse por tratarse de una masa de maíz, material muy frágil que se debe romper con un mordisco suave.? La

\begin{tabular}{|l|c|}
\hline \multicolumn{1}{|c|}{ VARIEDAD } & PEMDIEMTE \\
\hline Común & $0.26[0.0056]$ \\
\hline Híbrido A.M. & $0.028[0.0079]$ \\
\hline PabSA4 & $0.029[0.0091]$ \\
\hline QPM BLAMCO & $0.033[0.0054]$ \\
\hline QPM AMARILLO & $0.030[0.0063]$ \\
\hline
\end{tabular}

Clave: MC: maíz común; HAM: Híbrido AM; QPMB: QPM Blanco; QPMA: QPM Amarillo; PS4: PabSA4. Los valores entre corchetes se refieren a su desviación estándar

Tabla 7. Pendiente de la región elástica durante la compresión

variedad que presentó mayor fragilidad fue el MC y la menor fue QPMB, QPMA fue intermedio entre estos.

En la Fig. 2 se observan las curvas de las cinco muestras ensayadas. Se puede distinguir la semejanza en el comportamiento de las arepas de maíz HAM, PS4 y QPMA, las tres presentan una inclinación muy semejante en la región elástica (Tabla 7). ${ }^{4,6}$

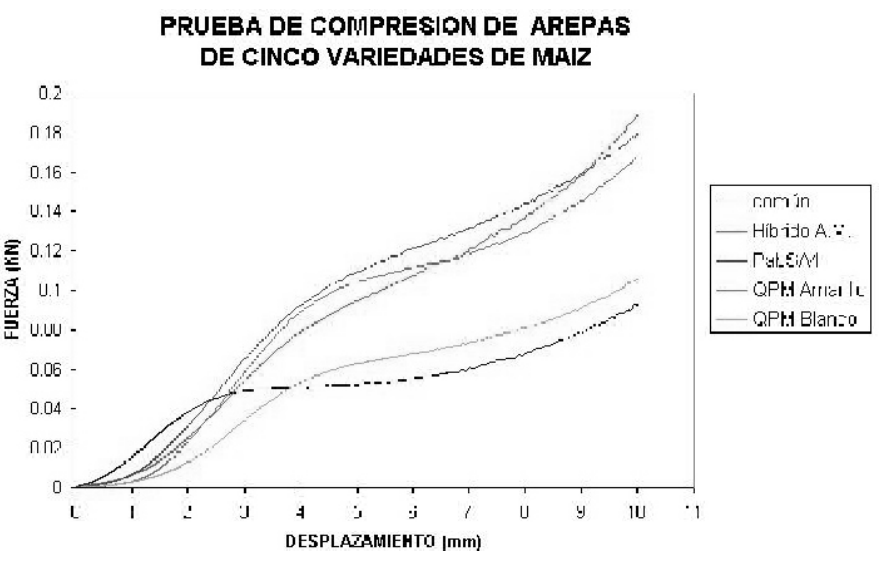

Figura 2. Curvas de compresión obtenidas en la Instron para las cinco variedades de maíz.

El punto de fluencia (donde termina la región elástica y se inicia la deformación permanente) es muy semejante en estas tres últimas variedades, mientras que en MC y QPMB son menores, implicando mayor fragilidad y menor fuerza para alcanzar el punto de compresión máxima. Esto indica que las arepas de MC y QPMB son más blandas, más fáciles de masticar que las arepas 
de las otras variedades, pero posiblemente más propensas a romperse durante el almacenamiento y el transporte. ${ }^{4,6,7}$

\section{Pruebas reológicas de extrusión de arepas sin asar}

En la Tabla 8 se reportan las pendientes de la región elástica obtenidas de las curvas de extrusión.

\begin{tabular}{|l|c|}
\hline \multicolumn{1}{|c|}{ VARIEDAD } & PEMDIEMTE \\
\hline Común & $0.20[0.0008]$ \\
\hline Híbrido A.M. & $0.020[0.0005]$ \\
\hline PabSA4 & $0.018[0.0007]$ \\
\hline QPM BLAMCO & $0.018[0.0006]$ \\
\hline QPM AMARILLO & $0.015[0.0007]$ \\
\hline
\end{tabular}

Clave: MC: maíz común; HAM: Híbrido AM; QPMB: QPM Blanco; QPMA: QPM Amarillo; PS4: PabSA4. Los valores entre corchetes se refieren a su desviación estándar

Tabla 8. Pendiente de la región elástica durante la extrusión

En la Figura 3 se observan las cinco curvas de extrusión correspondientes a las arepas de cada variedad de maíz. Se observa que las pendientes en la región elástica son muy semejantes entre sí, a pesar de la separación en tres grupos. Esta región lineal representa la compresión y la inclinación de esta línea se relaciona con la elasticidad, tomándose como un índice de firmeza. Esta parte de la curva es la transición del comportamiento de compresión a extrusión.

Luego, se observa un cambio en el comportamiento de la curva, la inclinación decae y es allí donde se presenta la ruptura de la arepa haciendo que fluya a través de la rejilla. Este punto representa el índice de cohesividad y representa la fuerza necesaria para comenzar el proceso de extrusión. ${ }^{4,6}$ Es a partir de este punto que la arepa comienza a extruirse, cambiando su inclinación y determinando la fuerza necesaria para continuar con el proceso. En esta parte la fuerza continúa incrementándose más lentamente, indicando que ocurren simultáneamente compresión, adhesión de la arepa a la celda de extrusión, extrusión y corte. . $^{6,7}$, El comportamiento de las cinco curvas es muy semejante, presentando características semejantes de firmeza, adhesividad y cohesividad. Sin embargo, debe profundizarse en el comportamiento mostrado por PS4 y QPMB, los cuales se apartan ostensiblemente de las otras variedades, indicando mayor adhesividad.

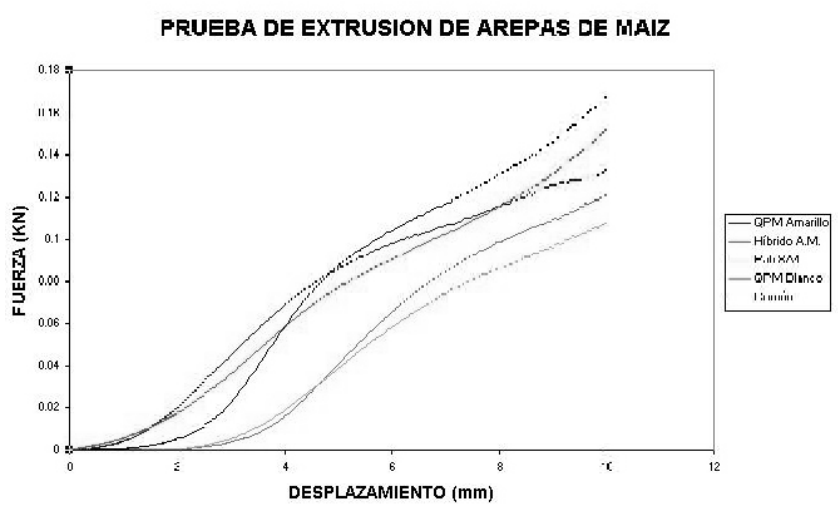

Figura 3. Curvas de extrusión obtenidas en la máquina Instron para las cinco variedades de maíz.

\section{CONCLUSIONES}

ఐ Todas las variedades de maíz estudiadas mostraron similar comportamiento durante el proceso de elaboración de arepas, aplicando un método estandarizado.

$\square$ El mejor método de preparación de arepas requirió 4 h de cocción y 17 h de remojo. Así, se obtuvo el mayor rendimiento durante la cocción y una masa más suave y manejable.

ఐ Los rendimientos después de la cocción siguieron el orden CM > QPMB > QPMA > HAM > PS4, mostrando la facilidad de absorción de agua de las variedades. Las arepas obtenidas de estas masas, perdieron entre 11 y $14 \%$ de humedad, después de asadas.

च El análisis químico mostró que el maíz QPM Blanco presentó semejanza con el maíz 
Características Físico-Químicas, Organolépticas y Reológicas de Arepas Procesadas a partir de Maíces de Alta Calidad Proteica (QPM)

común por su contenido de grasa y almidón semejantes. Hay diferencias en humedad y proteína, pues el común registra valores muy bajos comparado con el QPM Blanco, el cual es semejante a las otras variedades. Los maíces Híbrido A.M. y Pab SA4 se catalogaron como maíces altos en grasa, lo que le da un valor agregado a nivel comercial.

च El Híbrido AM y el QPM Amarillo fueron las variedades de mayor semejanza física con el maíz común (geométricas). Características que se pueden usar para establecer parámetros de diseño de silos de almacenamiento, equipo de procesamiento y para darle valor comercial al grano.

Tres de las cinco variedades, se clasificaron como Grado 3, correspondiente a maíz tipo exportación por sus medidas físicas.

Las arepas de las diferentes variedades de maíz tuvieron una buena aceptación organoléptica. Las muestras de mejor aceptación por su color, sabor y aroma fueron las de QPM Blanco y común, seguidas por QPM Amarillo, Híbrido A.M y PabSA4. Este último, presentó las más bajas puntuaciones en las tres pruebas; sin embargo, se considera un producto bueno dentro de la escala hedónica.

$\square$ La prueba reológica de compresión, mostró que las arepas de las cinco variedades analizadas, se catalogaron como productos frágiles. La inclinación de la curva en la sección elástica fue similar en todos los casos. También, se determinó que las arepas de maíz común y QPM Blanco eran las más blandas dentro del grupo, pues fueron las que menor fuerza requirieron para lograr la máxima compresión.

$\square$ Las curvas de extrusión de las arepas de las cinco variedades de maíz, se caracterizaron por poseer firmeza, cohesividad y adhesividad semejantes. Sin embargo, PS4 y QPMB mostraron comportamiento diferente a los demás y deben investigarse más.

\section{AGRADECIMIENTOS}

Los autores agradecen a LAMFOODS a través de la Dra. Jenny Ruales por proveer algunos fondos para soportar este trabajo y al Dr. Carlos Vélez, Departamento de Ingeniería de Alimentos de la Universidad del Valle por permitir el uso de la INSTROM para las pruebas reológicas.

\section{BIBLIOGRAFÍA}

1. American Association of Cereal Chemists. Standard Methods. Method 76-11. American Association of Cereal Chemists, St. Paul, Minn., USA. p. 1-4. 1995.

2. American Association of Cereal Chemists. Method 76-12, AACC, Minneapolis, USA, p.15. 1995.

3. American Association of Cereal Chemists. Method 73-13, AACC, Minneapolis, USA, p.16. 1995.

4. Askeland, R. La Ciencia y la Ingeniería de los Materiales. Grupo Iberoamericana, México. 1987.

5. Boyer, C. D. and Shannon, J. C. The use of endosperm genes for sweet corn improvement in: Plant Breeding Reviews, Vol. 1, Janick, J., Ed. AVI, Eastport, Conn., USA 1982.

6. De Man, J.M. Rheology and Texture in Food Quality. AVI, Eastport, Conn., USA 1986.

7. Hallauer, A. Specialty Corns. Department of Agronomy, lowa State University, Ames, lowa. 1994.

8. Lunven, P. El maíz en la nutrición humana. Organización de las Maciones Unidas para la Agricultura y la Alimentación, Roma. 1993.

9. Pedrero, F. y Pangborn, R. Evaluación sensorial de alimentos: métodos analíticos. Editorial Alhambra Mexicana, México. 1996.

10. Stanley, A.W. and Ramstad, P.E. Corn: Chemistry and Technology. American Association of Cereal Chemists, St. Paul, Minn., USA. 1994. 